\section{Efecto Antimicótico in vitro de Origanum vulgare sobre cepas de Candida albicans}

\author{
Antifungal effect in vitro of Origanum Vulgare in Candida Albicans \\ strains
}

\section{Resumen}

Objetivo: Evaluar el efecto antimicótico in vitro de aceite esencial de Origanum vulgare, sobre cepa de Candida albicans ATCC 10231 Material y método: Se efectuó la selección de cuatro de diferentes geotipos de orégano, se obtuvo los aceites esenciales mediante proceso de destilación por arrastre con vapor de agua; se determinó su composición química cuantitativa por Cromatografía de Gases, Espectrometría de Masas (CG/EM). Se evaluó in vitro la actividad antimicótica de los aceites esenciales a través de pruebas de sensibilidad con el método de difusión por discos en cultivo de agar frente a C. albicans.

Resultados: Los resultados mostraron alta actividad antimicótica de todas los aceites a partir de las concentración de 12,5\%. Conclusiones: Los aceites esenciales del Origanum vulgare de los diversos geotipos presentan diferencias en sus efectos antimicóticos. El aceite esencial de Origanum vulgare puede ser es una alternativa farmacológica para el tratamiento de infecciones micóticas en boca.

Palabras clave: Origanum vulgare, Candida albicans, antimicótico.

\section{Abstract}

Objective: To evaluate the in vitro antimycotic effect of Origanum vulgare essential oil on Candida albicans strain ATCC 10231 Material and method: Selection of four different oregano geotypes was carried out, the essential oils were obtained by distillation by Drag with steam; Its quantitative chemical composition was determined by Gas Chromatography, Mass Spectrometry (GC / MS). The antimicrotic activity of essential oils was evaluated in vitro through sensitivity tests with the disk diffusion method in agar culture against $C$. albicans. Results: The results showed high antimycotic activity of all oils from the concentration of $12.5 \%$. Conclusions: Essential oils of Origanum vulgare from different geotypes show differences in their antifungal effects. The essential oil of Origanum vulgare may be is a pharmacological alternative for the treatment of fungal infections in the mouth.

Keywords: Origanum vulgare, Candida albicans, Antifungal Agents.
Artículo Original

Jorge Eleodoro Villavicencio Gastelú, ${ }^{1}$ Hilda Moromi Nakata, ${ }^{3}$ Doris SalcedoMoncada, ${ }^{2}$ Martha Pineda-Mejía, ${ }^{2}$ Donald Ramos Perfecto, ${ }^{3}$ Livia Sonia Zambrano de la Peña, ${ }^{1}$ Elba Estefanía Martínez Cadillo, ${ }^{3}$ Gilberto Alejandro Mendoza Rojas, ${ }^{3}$ Marieta PetkovaGueorguieva, ${ }^{3}$ Roxana Margarita Bardales Álvarez ${ }^{4}$.

1. Departamento Médico Quirúrgico

2. Departamento de Estomatología Rehabilitadora

3. Departamento de Ciencias Básicas de la Facultad de Odontología de la Universidad Nacional Mayor de San Marcos

4. De la Universidad Nacional San Agustín de Arequipa

Correspondencia:

*Villavicencio Gastelú, Jorge Eleodoro

Correo electrónico: jvillavicenciog@unmsm.edu.pe Facultad de Odontología de UNMSM Av. Germán Amezaga 675. Lima 1, Perú

Coautores:

Hilda Moromi Nakat

hmnbio@hotmail.com

Doris Salcedo-Moncada

dsalcedom@unmsm.edu.pe

Martha Pineda-Mejía

mpinedam@unmsm.edu.pe

Donald Ramos Perfecto

dramos_37@hotmail.com

Livia Sonia Zambrano de la Peńa

facedent@hotmail.com

Elba Estefanía Martínez Cadillo emartinezc@unmsm.edu.pe

Gilberto Alejandro Mendoza Rojas amen807@hotmail.com

Marieta Petkova Guergoguieva mpgueorguieva@hotmail.com

Roxana Margarita Bardales Álvarez roxbaral@hotmail.com

\section{Introducción}

La candidiasis es una infección causada por hongos levaduriformes, de diferentes especies de este género. En el organismo se encuentra una gran variedad de microorganismos como bacterias y hongos, algunos son útiles y otros viven como saprofitos; sin embargo cuando el ecosistema del organismo o el sistema inmunitario se altera, éstos se desarrollan y provocan enfermedades oportunistas, se instalan principalmente en las superficies de las mucosas de la boca o vagina. ${ }^{1}$ Entre las levaduras del género Candida, Candida albicans, es la especie que comúnmente produce infecciones orales, comprendiendo hasta el $70 \%$ de los aislados, ${ }^{2}$ éstas son cada vez más prevalentes, especialmente en grupos de mayor riesgo. La resistencia de Candida sp. representa un reto terapéutico que deja un menor número de posibilidades para el tratamiento de estas infecciones que se caracterizan, a su vez, por una alta morbimortalidad. ${ }^{3}$ $\mathrm{El}$ aumento de infecciones oportunistas en la estomatitis subprotésica (ESP), principalmente en pacientes inmuno- 
comprometidos, la aparición de algunas cepas de microorganismos resistentes a los antimicrobianos y antimicóticos usualmente utilizados ha generado el interés en investigaciones dirigidas a los agentes fitoterapéuticos por las mínimas reacciones farmacológicas que presenta, teniendo incluso mayor potencial farmacológico que los productos de origen sintético. ${ }^{4}$

El orégano es una planta aromática originaria de Asia, muy utilizado en la actividad culinaria, cosmética y farmacéutica, contiene aceites esenciales, cuyos metabolitos secundarios como carvacrol y timol, tienen altos niveles de actividad antimicrobiana y antimicótica, siendo el timol el más activo. ${ }^{5,6}$ La composición química de los aceites esenciales y sus propiedades, están sujetos a variaciones debido a factores como el quimiotipo y el geotipo de la planta, por lo que es necesario su estudio para su correcta aplicación terapéutica.

Estudiando diferentes especies de orégano nativos de Europa y Méjico, encontraron que los rendimientos de extracción por el método de destilación con arrastre de vapor van desde el 1,8 al $5,6 \% .^{8}$

Un estudio de la eficacia de aceites esenciales contra diferentes micetos patogénicos evaluando in-vitro la inhibición de la actividad de alguno de estos y sus componentes principales fue realizado en contra de una cepa de Candida albicans. Dieciséis aceites esenciales comerciales, cuarenta y dos componentes puros (alcoholes, aldehídos, cetonas, fenoles e hidrocarburos), fueron probados mediante el uso de un agar semisólido. Los aceites esenciales de Origanum vulgare, Satureja montana, Mentha piperita, Cinnamomum verum, Cymbopogon flexuosus mostraron actividad inhibitoria máxima $(\mathrm{CMI}=500 \mathrm{ppm})$ después de 7 días. De acuerdo con los resultados del examen de los componentes puros, $\beta$-felandreno demostró ser el componente más interesante entre los hidrocarburos cíclicos monoterpénicos, ya que mostró fuerte actividad $(\mathrm{CMI}=50 \mathrm{ppm})$. El más activo de los fenoles fue carvacrol (CMI 100 ppm). ${ }^{5}$

En un estudio de orégano a partir de dos temperaturas de secado, 26 y 60 ${ }^{0} \mathrm{C}$; mencionan que en la composición química de los aceites esenciales, encontró presencia de carvacrol
$23,5 \%$ y $24,70 \%$ respectivamente, utilizado como alimento para ceba de conejos, señalan su acción bactericida y bacteriostática. No menciona haber encontrado timol. ${ }^{9}$

Otro estudio evaluó la actividad in vitro de los aceites esenciales extraídos de canela, orégano mexicano, tomillo y jengibre contra algunas especies de Candida resistentes y susceptibles a fluconazol que fueron aislados de pacientes con VIH avanzado y afectados por resistencia al fluconazol o inmunocomprometidos con infecciones fúngicas diseminadas. Los resultados mostraron que el aceite esencial de orégano tuvo mayor actividad antifúngica en todas las cepas de Candida incluyendo la Candida tropicalis y Candida krusei. El aceite de orégano mexicano en casi todas las cepas a excepción de la Candida tropicalis y el aceite esencial de jengibre fue el de más baja actividad antifúngica. La Candidiasis orofaríngea se desarrolla en 80 y $95 \%$ de los pacientes con SIDA. Entre los componentes químicos encontrados por (CG/ EM) el orégano mexicano mostró timol $20,17 \%$ y carvacrol $56.8 \% .^{10}$

Las propiedades antimicrobianas del aceite esencial de orégano in vitro fueron estudiados para determinar su eficacia en la inhibición del crecimiento de levaduras: Candida albicans, Cryptococcus albidus, Cryptococcus neoformans y Rhodotorula rubrum; mediante medios líquido suplementado con 200 y 400 $\mu \mathrm{g} / \mathrm{mL}$ de aceite esencial de orégano a $37^{\circ} \mathrm{C}$ durante $24,40,60$ y 85 horas; el efecto inhibidor del aceite esencial del orégano por el método de difusión en disco, fue de un diámetro de $27 \mathrm{~mm}$ del crecimiento de las levaduras. La mayor duración de incubación resulto en un mayor porcentaje de inhibición del crecimiento de las cepas, concluyendo que la actividad antimicrobiana del aceite esencial del orégano es fungistático y fungicida; así mismo, encontró que la CMI es $20 \mu \mathrm{g} / \mathrm{mL}$.

\section{Materiales y método:}

\section{Extracción de aceites esenciales:}

Se recolectó de distintos lugares del Perú, cuatro muestras de diferentes especies de orégano, considerando que por el geotipo, presentarían diferente quimiotipo: ${ }^{8,11}$ el Origanum vulgare $\mathrm{L}$ (Jauja y Tacna), Origanum $x$ intercedens y Origanum $x$ majoricum (Arequipa). La clasificación taxonómica fue realizada en el Laboratorio del Herbario San
Marcos (USM) del Museo de Historia Natural, de la Universidad Nacional Mayor de San Marcos

El proceso de extracción de aceites esenciales de orégano, se realizó utilizando la técnica de destilación por arrastre con vapor de agua, a partir de las hojas y flores de plantas frescas y semideshidratadas; el aceite fue recolectado y decantado en un vaso florentino; la deshidratación del aceite obtenido se efectuó utilizando sulfato de sodio anhidro $\left(\mathrm{SO}_{4} \mathrm{Na}_{2}\right)$ y se obtuvo un rendimiento promedio de $0,546 \mathrm{~mL}$ de aceite $/ 100 \mathrm{~g}$ de hojas y flores.

\section{Composición química de los aceites esenciales}

El estudio de la composición química del aceite esencial de orégano se realizó sometiendo a la muestra a un análisis por Cromatografía de Gases/ Espectrometría de masas (CG/EM). La muestra: se diluyó $20 \mu \mathrm{L}$ de aceite esencial en $1 \mathrm{ml}$ de diclorometano. La identificación de los compuestos se realizó utilizando los índices de retención de Kovats, usando una serie de n-alcanos (C6-C32) y mediante comparación de los espectros de masas obtenidos con aquellos de la librería de espectros Wiley. La cuantificación se realizó mediante el cálculo del porcentaje relativo de áreas de cada compuesto.

Para el análisis estadístico se tuvo en cuenta únicamente el porcentaje relativo de timol como componente mayoritario y de interés para el presente estudio por sus propiedades antioxidantes y antimicrobianas. ${ }^{12}$

\section{Efecto antimicótico de los aceites esenciales sobre C. albicans cepa ATCC 10231}

El estudio fue experimental in vitro, prospectivo, transversal, se analizó en cultivos de la cepa ATCC de Candida, en Agar Sabouraud Glucosado y sometidas a la acción de cuatro tipos de aceite esencial de orégano por el método de difusión mediante discos de papel Whatman $\mathrm{n}^{\circ} 1$ de $6 \mathrm{~mm}$ de diámetros, embebidos en concentraciones de 12,5 \%, $25 \%, 50 \%$ y $100 \%$, utilizándose como control positivo Miconazol (Daktarin ${ }^{\circledast}$ gel oral $20 \mathrm{mg} / \mathrm{g}$ ); como control Dimetilsulfóxido (DMSO).

Para el análisis de los resultados se consideró los valores de sensibilidad según el método de aromatograma de Duraffoourd ${ }^{13,14}:{ }^{1} \mathrm{~S}$. nula: $\leq 8 \mathrm{~mm},{ }^{2} \mathrm{~S}$. límite 
9-14 mm, ${ }^{3} \mathrm{~S}$. media $15-19 \mathrm{~mm}$ y ${ }^{4} \mathrm{~S}$. sumamente sensible $\geq 20 \mathrm{~mm}$.

\section{RESULTADOS}

Rendimiento de aceite essencial por especie

Tabla 1. Rendimiento de aceite esencial

\begin{tabular}{|c|c|c|c|c|c|}
\hline $\begin{array}{c}\text { Proce- } \\
\text { dencia }\end{array}$ & $\begin{array}{c}\text { Muestra } \\
\text { (estado) }\end{array}$ & $\begin{array}{c}\text { Hojas } \\
\text { f flores } \\
\text { (peso en } \\
\mathrm{Kg} .)\end{array}$ & $\begin{array}{c}\text { Rendi- } \\
\text { miento } \\
\text { total }(\mathrm{mL} \text { ) }\end{array}$ & $\begin{array}{c}\text { Rendi- } \\
\text { miento } \\
\text { (mL/ } \\
\mathrm{Kg})\end{array}$ & $\begin{array}{c}\text { Volu- } \\
\text { men/ } \\
\text { Peso } \\
(\%)\end{array}$ \\
\hline $\begin{array}{c}\text { Origanum } \\
\text { vulgare L } \\
\text { (Jauja) }\end{array}$ & Fresco & $1,600 \mathrm{Kg}$ & $5,3 \mathrm{~mL}$ & 3.31 & 0,22 \\
\hline $\begin{array}{c}\text { Origanum } \\
\text { vulgare L } \\
\text { (Tacna) }\end{array}$ & Fresco & $3,100 \mathrm{Kg}$ & $15 \mathrm{~mL}$ & 4,83 & 0,48 \\
\hline $\begin{array}{c}\text { Origanum x } \\
\text { intercedens } \\
\text { (Arequipa) }\end{array}$ & $\begin{array}{c}\text { Deshidra- } \\
\text { tado }\end{array}$ & $1,955 \mathrm{~kg}$ & $30 \mathrm{~mL}$ & 15.34 & 1.53 \\
\hline $\begin{array}{c}\text { Origanum x } \\
\text { majoricum } \\
\text { (Arequipa) }\end{array}$ & $\begin{array}{c}\text { Deshidra- } \\
\text { tado }\end{array}$ & $1,985 \mathrm{Kg}$ & $45 \mathrm{~mL}$ & 22.67 & 2.27 \\
\hline
\end{tabular}

\section{Composición química}

La composición química del aceite esencial, al análisis por Cromatografía de Gases/ Espectrometría de masas (CG/EM) de cada especie de orégano estudiado:

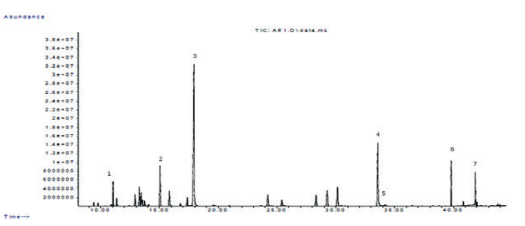

Figura 1. Cromatograma de gases de aceite esencial Origanum $\mathbf{x}$ intercedens (Arequipa).-

o-cimeno (1), y-terpineno (2), cis- $\beta$ terpineol (3), timol (4), carvacrol (5), $\beta$-cariofileno (6) y elixeno (7).

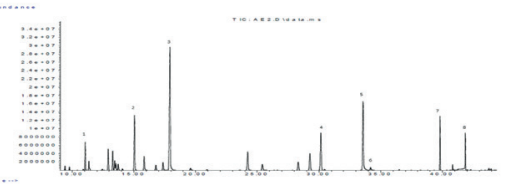

Figura 2. Cromatograma de gases de aceite esencial Origanum x majoricum (Arequipa)

sabineno (1), a terpineno y o-cimeno se ubican antes del $\alpha$ terpineno (2), cis$\beta$-terpineol (3), acetato de linalol (4), timol (5), carvacrol (6), $\beta$-cariofileno (7) y y-elemeno (8).

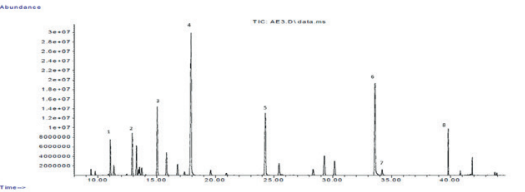

Figura 3. Cromatograma de gases de aceite essencial Origanum vulgare $L$ (Tacna).
Sabineno (1), $\alpha$ terpineno (2), y-terpineno (3) cis- $\beta$-terpineol (4), 4-terpineol (5), timol (6), carvacrol (7), $\beta$-cariofileno (8)

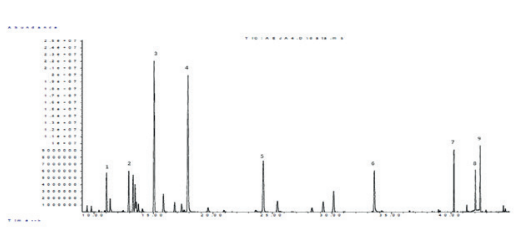

Figura 4. Cromatograma de gases de aceite esencial Origanum vulgare $L$ (Jauja)

Sabineno (1), $\alpha$ terpineno y p-cimeno (2), $\gamma$-terpineno (3) cis- $\beta$-terpineol (4), 4-terpineol (5), timol (6), carvacrol (no figura), $\beta$-cariofileno (7), $\beta$-cubebeno (8) y y-elemeno (9).

\section{Actividad antimicótica}

La actividad antimicótica de los aceites esencial del orégano sobre Cándida albicans ATCC, se determinó en función al diámetro de halo de inhibición y los criterios del método de arotmatorgrama de Duraffoourd así como de la concentración mínima inhibitoria (HCMI). ${ }^{13}$ Tabla 2, 3 y 4 . A la concentración de $12,5 \%$, Origanum $x$ majoricum (Arequipa), presenta mayor actividad antimicótica sobre Candida albicans ATCC.

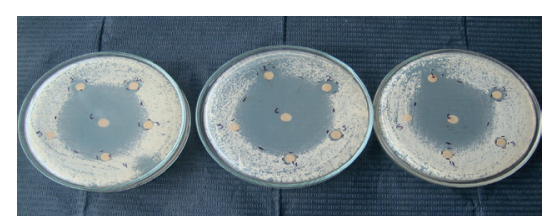

Figura 5. Actividad antimicótica de los aceites esencial del orégano sobre Cándida albicans ATCC.

Tabla 2. Sensibilidad antimicótica de los aceites esenciales de cuatro especies de Orégano sobre Candida albicans cepa ATCC 10231

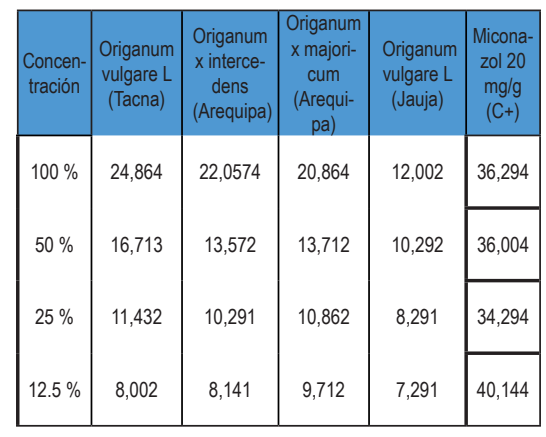

Valores de sensibilidad en el aromatograma según Duraffourd ${ }^{13}$

${ }^{1}$ S. nula: $\leq 8 \mathrm{~mm}$; ${ }^{2}$ S. límite: 9-14 mm; ${ }^{3} \mathrm{~S}$. media: $15-19 \mathrm{~mm}$; ${ }^{4}$ Sumamente sensible: $\geq 20 \mathrm{~mm}$.
Tabla 3. Efectividad antimicótica determinados por promedio de halo de inhibición de aceites esenciales de orégano, control C (+) Miconazol 20 $\mathrm{mg} / \mathrm{g}$, sobre Candida albicans ATCC 10231

\begin{tabular}{|c|c|c|c|c|}
\hline \multirow[t]{2}{*}{ Concentración } & \multirow[t]{2}{*}{ Orégano } & \multicolumn{3}{|c|}{$\begin{array}{l}\text { Halo de inhibición (mm } \\
\text { del diámetro) }\end{array}$} \\
\hline & & media & D.E. & \begin{tabular}{|l} 
P. Valor \\
\end{tabular} \\
\hline \multirow{5}{*}{$100 \%$} & \multirow{5}{*}{$\begin{array}{c}p \text { valor } \\
\text { Origanum vulgare } L \text { (Tacna) } \\
\text { Origanum } x \text { intercedens } \\
\text { (Arequipa) } \\
\text { Origanum x majoricum } \\
\text { (Arequipa) } \\
\text { Origanum vulgare } L \text { (Jauja) }\end{array}$} & & & \multirow{5}{*}{0,000} \\
\hline & & 24,86 & 6,67 & \\
\hline & & 22,57 & 2,94 & \\
\hline & & 20,86 & 5,11 & \\
\hline & & 12,00 & 1,63 & \\
\hline \multirow[t]{4}{*}{$50 \%$} & Origanum vulgare L (Tacna) & 16,71 & 4,23 & \multirow[t]{4}{*}{0,001} \\
\hline & \multirow{3}{*}{$\begin{array}{c}\text { Origanum } x \text { intercedens } \\
\text { (Arequipa) } \\
\text { Origanum x majoricum } \\
\text { (Arequipa) } \\
\text { Origanum vulgare L (Jauja) }\end{array}$} & 13,57 & 1,51 & \\
\hline & & 13,71 & 1,50 & \\
\hline & & 10,29 & 1,25 & \\
\hline \multirow[t]{4}{*}{$25 \%$} & \multirow{4}{*}{$\begin{array}{l}\text { Origanum vulgare } \mathrm{L} \text { (Tacna) } \\
\text { Origanum } \mathrm{x} \text { intercedens } \\
\text { (Arequipa) } \\
\text { Origanum x majoricum } \\
\text { (Arequipa) } \\
\text { Origanum vulgare L (Jauja) }\end{array}$} & 11,43 & 1,13 & \multirow[t]{4}{*}{0,000} \\
\hline & & 10,29 & 0,49 & \\
\hline & & 10,86 & 1,34 & \\
\hline & & 8,43 & 1,81 & \\
\hline \multirow[t]{4}{*}{$12.5 \%$} & Origanum vulgare L (Tacna) & 8,00 & 0,82 & \multirow[t]{4}{*}{0,005} \\
\hline & \multirow{3}{*}{$\begin{array}{c}\text { Origanum x intercedens } \\
\text { (Arequipa) } \\
\text { Origanum x majoricum } \\
\text { (Arequipa) } \\
\text { Origanum vulgare L (Jauja) }\end{array}$} & 8,14 & 1,22 & \\
\hline & & 9,71 & 1,11 & \\
\hline & & 7,29 & 1,38 & \\
\hline \multirow{4}{*}{$\begin{array}{c}\text { Miconazol } \\
20 \mathrm{mg} / \mathrm{g}\end{array}$} & \multirow{4}{*}{$\begin{array}{l}\text { Origanum vulgare } L \text { (Tacna) } \\
\text { Origanum x intercedens } \\
\text { (Arequipa) } \\
\text { Origanum x majoricum } \\
\text { (Arequipa) } \\
\text { Origanum vulgare L (Jauja) }\end{array}$} & 38,57 & & \\
\hline & & 38,00 & & \\
\hline & & 27,43 & & \\
\hline & & 41,29 & & \\
\hline
\end{tabular}

Tabla 4. Actividad antimicótica de aceites esenciales de orégano, control

C (+) Miconazol, sobre Candida

albicans ATCC 10231. (Prueba ANOVA)

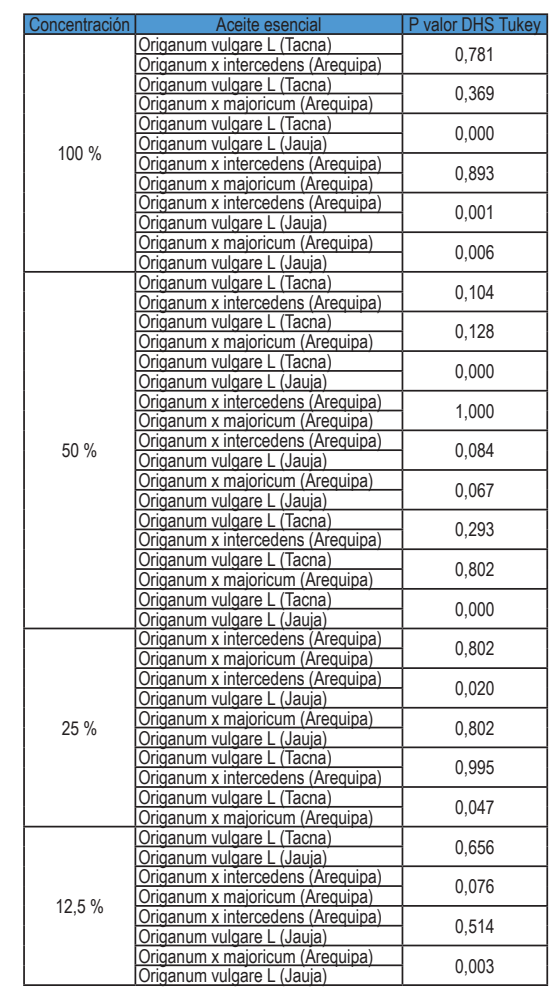




\section{DISCUSIÓN}

La obtención del de aceite esencial de orégano con la técnica de destilación por arrastre con vapor de agua arrojó rendimiento entre $0,22 \%$ al $2,27 \%$, a diferencia de los valores mostrados por Arcila-Lozano ${ }^{8}$ fue entre $1,8 \%$ al 5,6 \%

En los aceites esenciales Origanum $x$ intercedens, Origanum $x$ majoricum (Arequipa) y Origanum vulgare $L$ (Tacna, Jauja), se identificó por Cromatografía de Gases-Espectrometría de Masas (CG/EM), más de treinta componentes químicos, El más importante el timol en concentraciones de $5,86 \%$ a $14,71 \%$ y carvacrol entre $0,53 \%$ a $0,88 \%$. No encontrándose en Origanum vulgare L (Jauja); Arcila-Lozano ${ }^{8}$ afirma que encontró contenidos de timol superiores al $30 \%$. mientras que Ayala ${ }^{9}$ encontró carvacrol entre $23,5 \%$ a $24,70 \%$. Tampieri ${ }^{5}$ indican que $\beta$-felandreno es el componente con altos niveles de actividad antimicrobiana;

El aceite esencial de orégano presentó propiedad antimicótica para Candida albicans ATCC 10231, cuya concentración mínima inhibitoria (HCMI) fue 12,5 \% y sumamente sensible al $100 \%$ con $24,86 \mathrm{~mm}$ de diámetro; Adams $^{7}$ menciona que la acción del aceite esencial in vitro sobre Candida albicans y otras levaduras mediante medios líquido suplementado con 200 y $400 \mu \mathrm{g} / \mathrm{mL}$ de aceite esencial de orégano a $37^{\circ} \mathrm{C}$ durante 24,40 , 60 y 85 horas fue de un diámetro de $27 \mathrm{~mm}$. Arcila-Lozano ${ }^{8}$ menciona que para Listeria multiflora y Listeria chevalieri, los valores de HCMI fue $0,65 \mathrm{mg} / \mathrm{ml}$ a1,27 $\mathrm{mg} / \mathrm{ml}$ para hongos debido a su alto contenido de timol y precursores biosintéticos y-terpineno y p-cimeno; Tampieri ${ }^{5}$ demuestra en su estudio con aceites esenciales comerciales, actividad inhibitoria máxima es igual a 500 ppm después de 7 días

\section{CONCLUSIONES}

1. Se determinó que el aceite esencial de Origanum vulgare L, posee efecto antimicótico sobre Candida albicans cepa ATCC 10231 en concentraciones de $100 \%, 50 \%$ $25 \%$ y $12,5 \%$
2. Los aceites esenciales del Origanum vulgare $\mathrm{L}$ de los diversos geotipos presentan diferencias en sus efectos antimicóticos. siendo el de Origanum vulgare L (Tacna) el más efectivo.

3. EL aceite esencial de Origanum vulgare $\mathrm{L}$,es antimicótico con potencia farmacológica, siendo una alternativa para tratamiento de la candidiasis

\section{Referencias bibliográficas}

1. University of Maryland Medical Center. Candidiasis bucal [Internet].. [citado 10 de diciembre de 2016]. Disponible en: http:// umm.edu/health/medical/spanishency /articles/candidiasis-bucal

2. Lee SP, Buber MT, Yang Q, Cerne R, Cortés RY, Sprous DG, et al. Thymol and related alkyl phenols activate the hTRPA1 channel. Br J Pharmacol. abril de 2008;153(8):1739-49.

3. Dhanasekaran D, Vinothini K, Latha S, Thajuddin N, Panneerselvam A. Human dental biofilm: Screening, characterization, in vitro biofilm formation and antifungal resistance of Candida spp. Saudi J Dent Res. enero de 2014;5(1):55-70.

4. Cornely OA, Bassetti M, Calandra T, Garbino J, Kullberg BJ, Lortholary $\mathrm{O}$, et al. ESCMID* guideline for the diagnosis and management of Candida diseases 2012: nonneutropenic adult patients. Clin Microbiol Infect Off Publ Eur Soc Clin Microbiol Infect Dis. diciembre de 2012;18 Suppl 7:19-37.

5. Tampieri MP, Galuppi R, Macchioni F, Carelle MS, Falcioni L, Cioni PL, et al. The inhibition of Candida albicans by selected essential oils and their major components. Mycopathologia. 1 de abril de 2005;159(3):339-45.

6. da Silva FC, Majewski M, Yamamoto LT, Cardoso Jorge AO, Koga-Ito CY. Atividade Antimicrobiana Da Tintura E Infusão De Origanum Vulgare (Orégano). (Portuguese). Rev Salusvita. diciembre de 2008;27(3):353-61.

7. Adams A, Kumar S, Clauson M, Sahi S. Anti-yeast activities of Origanum oil against human pathoge- nic yeasts. Adv Biosci Biotechnol. 2011;2(2):103-7.

8. Arcila-Lozano CC, Loarca-Piña G, Lecona-Uribe S, González de Mejía E. [Oregano: properties, composition and biological activity]. Arch Latinoam Nutr. marzo de 2004;54(1):100-11.

9. Ayala L, Silvana N, Zocarrato I, Gómez S. Utilización del orégano vulgar (Origanum vulgare) como fitobiótico en conejos de ceba. (Spanish). Rev Cuba Cienc Agric. mayo de 2011;45(2):159-61.

10. Pozzatti P, Scheid LA, Spader TB, Atayde ML, Santurio JM, Alves $\mathrm{SH}$. In vitro activity of essential oils extracted from plants used as spices against fluconazole-resistant and fluconazole-susceptible Candida spp. Can J Microbiol. noviembre de 2008;54(11):950-6.

11. Baranska M, Schulz H, Krüger H, Quilitzsch R. Chemotaxonomy of aromatic plants of the genus Origanum via vibrational spectroscopy. Anal Bioanal Chem. 15 de febrero de 2005;381(6):1241-7.

12. De Castro RD, de Souza TMPA, Bezerra LMD, Ferreira GLS, de Brito Costa EMM, Cavalcanti AL. Antifungal activity and mode of action of thymol and its synergism with nystatin against Candida species involved with infections in the oral cavity: an in vitro study. BMC Complement Altern Med [Internet]. 24 de noviembre de 2015 [citado 2 de noviembre de 2016];15. Disponible en: http:// www.ncbi.nlm.nih.gov/pmc/articles/PMC4659158/

13. Alzamora Libertad. Medicina Tradicional en el Perú: Actividad Antimicrobiana in vitro de los Aceites Esenciales Extraídos de Algunas Plantas Aromáticas. Anales de la Facultad de Medicina Universidad Nacional Mayor de San Marcos. 2001;62,Nro.2:156-61.

14. García López A, Vizoso Parra Á, Ramos Ruiz A, Piloto J. Estudio toxicogenético de un extracto fluido de ocimun basilicum 1. (albahaca blanca). Rev Cuba Plantas Med. diciembre de 2000;5(3):78-83. 